\title{
Environmental issues due to organohalogenated compounds diffuse pollution in groundwater. Emerging issue in the Roman area?
}

\section{Criticità ambientali per contaminazione diffusa da organo alogenati nelle acque di falda. Problematica emergente nella realtà romana?}

\author{
Isidoro Bonfà, Francesco La Vigna, Simona Martelli, Lucilla Ticconi
}

\begin{abstract}
Riassunto: La maggior parte dei grandi centri abitati, specialmente quelli caratterizzati da una forte vocazione industriale, presentano importanti situazioni di contaminazione delle acque di falda da composti organo alogenati. Anche nella Città di Roma, sebbene presenti uno sviluppo industriale molto limitato, negli ultimi anni sono stati notificati alcuni siti con questo tipo di contaminazione. Questi siti sono prevalentemente distribuiti nel settore orientale della città dove sono anche concentrate le poche attività industriali presenti. I valori di concentrazione dei contaminanti coprono un vasto range e mostrano sia casi di chiara contaminazione puntuale, dove i valori sono molto alti, sia casi da cui è possibile ipotizzare una probabile contaminazione da sorgenti multiple diffuse. I siti notificati ricadono in un settore cruciale per l'idrogeologia di Roma, ovvero in corrispondenza dell'acquifero posto all'interno del "Graben del Paleotevere" molto sfruttato per diversi usi. Sebbene ad oggi non sussista una accertata contaminazione di origine diffusa da composti organo alogenati a Roma, lo scopo di questo lavoro è solo quello di inquadrare, a livello preliminare e sulla base dei dati disponibili, questa problematica emergente.
\end{abstract}

Parole chiave: acque sotterranee, valori di fondo antropico, solventi clorurati, PCE, TCE, Roma, Italia.

Keywords: groundwater, background pollution values, chlorinated solvents, PCE, TCE, Rome, Italy.

Francesco LA VIGNA 童”

Roma Capitale - Dipartimento Tutela Ambientale

Servizio Bonifica dei siti inquinati

Geologia Ambientale e Inquinamento Diffuso

francesco.lavigna@comune.roma.it

Isidoro BONFÀ

Simona MARTELLI

Lucilla TICCONI

Roma Capitale - Dipartimento Tutela Ambientale

Servizio Bonifica dei siti inquinati

Geologia Ambientale e Inquinamento Diffuso

Ricevuto/Received: 26 June 2017-Accettato/Accepted: 28 June 2017 Pubblicato online/Published online: 30 June 2017

This is an open access article under the CC BY-NC-ND license: http://creativecommons.org/licenses/by-nc-nd/4.0/

(C) Associazione Acque Sotterranee 2017

\begin{abstract}
Most of the large urban areas, especially those with a strong industrial vocation, present important contamination of organoblaogenated compunds. Even in the City of Rome, although it has a very limited industrial development, some sites interested by this kind of contamination have been notified in recent years. The distribution of these sites is prevalent in the eastern sector of the city where the few industrial activities present are also located. Concentrations are varied and show both cases of clear point source contamination, where values are very high, and cases where a possible contamination from multiple point sources can be hypothesized. The sites reported fall into a crucial area for the hydrogeology of Rome, that is the "Paleotiber Graben" aquifer, exploited for various uses. To date, there is no proven diffuse contamination of organohalogenated compounds in Rome, nevertheless the purpose of this work is to frame this emerging issue on the basis of the available data.
\end{abstract}

\section{Introduzione}

La relazione tra tessuto urbano e acque sotterranee comporta inevitabilmente degli impatti sia sul naturale deflusso sotterraneo sia sulla qualità di queste (Ellis 1999). Le opere infrastrutturali urbane possono infatti influenzare le acque sotterranee sia fisicamente per via di effetti barriera, che da un punto di vista della ricarica per via delle perdite dei sistemi di adduzione e smaltimento delle acque, determinando variazioni del campo di moto (Howard and Israfilow 2002, VazquèzSuňè et al 2005). La presenza concentrata di diverse attività industriali nonché commerciali, tra cui numerose officine e lavanderie ma anche moltissimi punti vendita carburante, può determinare nel tempo locali sversamenti accidentali o talvolta dolosi di sostanze inquinanti che infiltrandosi direttamente nel terreno o disperdendosi dalle reti fognarie, quasi mai completamente sigillate, danno luogo a fenomeni di contaminazione dei terreni e delle falde acquifere, in origine puntuali, ma che con il passare del tempo e in base alle velocità di deflusso sotterraneo e alle caratteristiche idrodinamiche degli acquiferi interessati, possono assumere carattere diffuso in falda.

I siti potenzialmente contaminati censiti nella città di Roma sono riconducibili, per tipologia di fonte, essenzialmente a due grandi categorie: la prima comprende siti inquinati da fonti localizzate e la seconda siti che presentano alterazioni di fondo di origine naturale (La Vigna et al. 2014, Preziosi et al. 2009), con l'eccezione di un certo numero di siti la cui fonte di contaminazione resta tuttora da chiarire da parte della Città Metropolitana di Roma Capitale, Ente preposto alle indagini. 
Con riguardo alla prima categoria, la maggior parte degli episodi di contaminazione a Roma deriva da fonti localizzate: linee di distribuzione e serbatoi interrati o fuori terra, sversamenti accidentali, abbandono di rifiuti, attività commerciali o industriali comunemente diffuse su tutto territorio urbanizzato. Ad oggi i siti notificati sul territorio della città di Roma dal 1999, molti dei quali riguardanti episodi di potenziale contaminazione del solo suolo e non delle acque di falda, sono circa 350, solo in parte bonificati e certificati o autocertificati (Fig.1).

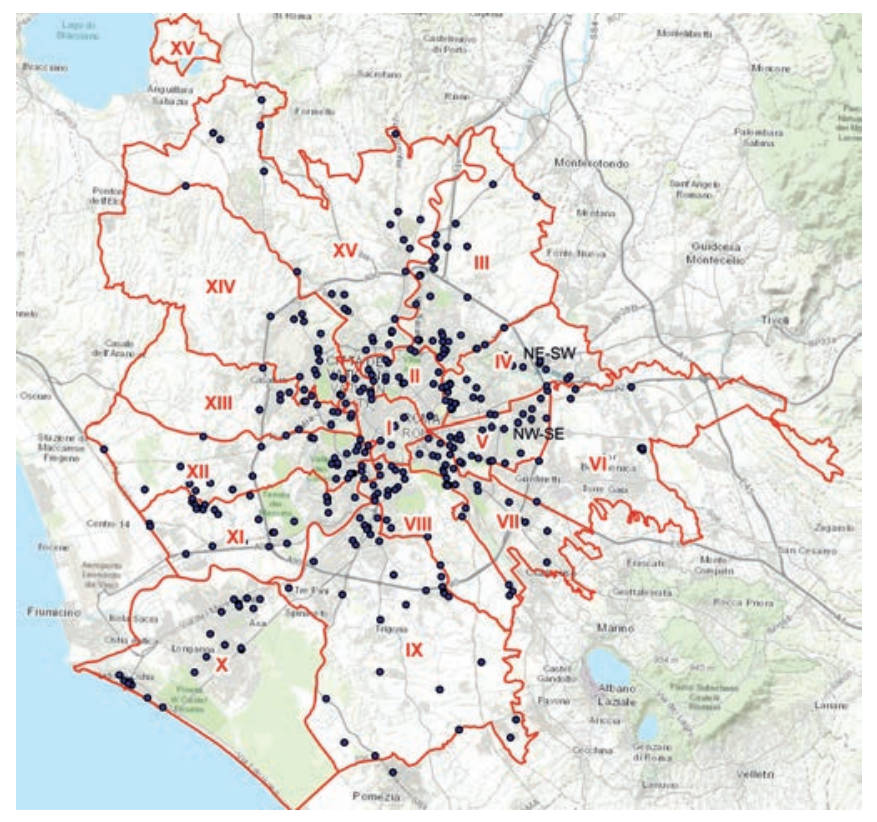

Fig. 1 -Distribution of the notified contaminated sites in Rome. Only part of those are also related to groundwater.

Fig. 1 - Distribuzione dei siti contaminati notificati a Roma. Solo parte di questi interessano anche le acque sotterranee.

Predominano numericamente i casi di contaminazione da sostanze di origine idrocarburica derivanti da perdite della rete dei punti vendita carburanti. In secondo ordine, ma non per rilevanza dei fenomeni, sono presenti diversi casi di contaminazione della falda da composti clorurati (DNAPL) soprattutto nel quadrante Est della città e notificati di recente. Inoltre è importante richiamare la possibilità di solubilizzazione di sostanze normalmente poco solubili (come taluni metalli) indotta dalla contaminazione antropica, nel momento in cui quest'ultima determina variazioni del $\mathrm{pH}$ e delle condizioni redox locali. In tali situazioni fenomeni di contaminazione secondaria da metalli possono sovrapporsi alle alterazioni di carattere naturale degli stessi, complicando il quadro ambientale del sito ed introducendo criticità gestionali nell'ambito dei procedimenti amministrativi di bonifica.

Tra i più comuni tipi di DNAPL si possono includere composti che sono stati per anni impiegati largamente nei processi industriali come $i$ solventi clorurati (es. Tetracloroetilene e Tricloroetilene), impiegati principalmente come sgrassanti.

Sebbene tali sostanze siano state prodotte e utilizzate sin dall'inizio del secolo scorso, la loro importanza come contaminanti delle acque di falda è stata riconosciuta soltanto a partire dall'inizio del 1980; ciò è da imputare alla mancanza di una legislazione che potesse prevenire lo sversamento incontrollato di contaminanti nel sottosuolo, e all'assenza di metodi analitici in grado di rilevare questi composti anche a basse concentrazioni. Bisogna aggiungere inoltre che molti manuali di sicurezza delle industrie chimiche, distribuiti tra il 1940 e il 1980, suggerivano come metodo di smaltimento di solventi clorurati il loro spargimento in terreni secchi per permettere la relativa evaporazione, non tenendo conto della capacita dei DNAPL di infiltrarsi rapidamente nel terreno, provocando quindi una contaminazione del suolo e delle porzioni profonde degli acquiferi (Verdini and Majone 2015).

I solventi clorurati sono contaminanti molto frequenti nei contesti antropizzati e specialmente in quelli a forte vocazione industriale. In Italia vi sono casi molto noti come quelli, ad esempio, in Lombardia, Piemonte, Veneto e Toscana (Alberti et al. 2017, Busoni et al. 2013, Guzzella et al. 2009, Menichetti and Doni 2017, Pedretti et al. 2013, Vanzetti et al. 2016) dove la contaminazione, sebbene si sia originata da fonti puntuali, oggi presenta carattere diffuso, anche per la molteplicità delle sorgenti.

Lo scopo di questo lavoro è quello di inquadrare, per quanto a livello preliminare e sulla base dei soli dati in possesso dell'Amministrazione Capitolina, la problematica emergente a Roma relativa alla contaminazione da sostanze organo alogenate nelle acque di falda con particolare riferimento al settore Est della città.

\section{Inquadramento idrogeologico}

L'assetto idrogeologico e lo schema di circolazione delle acque sotterranee nel territorio di Roma sono stati recentemente descritti nella Carta Idrogeologica di Roma in scala 1:50.000 (La Vigna and Mazza 2015) e anche nei lavori di Mazza et al. (2016), La Vigna et al. (2008,2016), Capelli et al. (2008) e Ventriglia (1971, 2002). L'idrogeologia del territorio di Roma Capitale è condizionata dall'assetto strutturale, dai rapporti di scambio idrico tra cinque diverse Unità Idrogeologiche e dalla presenza di due importanti corsi d'acqua perenni. Le depressioni e le elevazioni strutturali, unitamente alle differenti permeabilità che caratterizzano alcuni principali complessi idrogeologici, determinano i limiti tra i grandi acquiferi e le linee di flusso delle acque sotterranee in essi presenti (Mazza et al, 2016).

Nel territorio di Roma Capitale si incontrano domini geologici diversi, che danno luogo a cinque Unità Idrogeologiche:

- Unità Idrogeologica dei Monti Sabatini;

- Unità Idrogeologica dei Colli Albani;

- Unità Idrogeologica dei depositi continentali prevulcanici;

- Unità Idrogeologica dei depositi alluvionali recenti e attuali;

- Unità Idrogeologica del Delta del Fiume Tevere.

I molteplici acquiferi presenti in queste idrostrutture hanno una circolazione complessa che tende a raccordarsi con 
le quote dei livelli di base fondamentali, costituiti dai fiumi Tevere, Aniene ed il mare (Fig.2).

Le Unità Idrogeologiche sono limitate inferiormente dal substrato a bassa permeabilità costituito da un Complesso argilloso-sabbioso basale (formazione di Monte Vaticano, di Monte delle Piche e dai livelli inferiori della formazione di Monte Mario) che funge da acquicludo.

Gli acquiferi vengono in parte ricaricati anche da aree esterne all'ambito territoriale rappresentato in carta. Il tetto del Complesso argilloso-sabbioso basale presenta una superficie fortemente articolata, derivante dalle condizioni morfo-strutturali e dall'azione di modellamento del reticolo idrografico antecedente alla messa in posto dei depositi vulcanici (Dimasi et al. 2015). Dall'andamento di detta superficie (Fig.3) si possono notare due incisioni principali; la prima è costituita da una depressione controllata da tettonica ad andamento "appenninico" collocata in corrispondenza dei settori orientali della Città e chiamata dagli autori "Graben del Paleotevere" (Luberti et al. 2017), la seconda è costituita dall'incisione del Fiume Tevere avvenuta durante un periodo di basso stazionamento marino durante l'ultima era glaciale (fase Würmiana). La circolazione delle acque sotterranee, negli acquiferi maggiormente profondi, è ovviamente influenzata dalla presenza di questo complesso a bassissima permeabilità.

Nell'area romana sono state individuate differenti circolazioni sotterranee. In particolare, oltre ad una circolazione "regionale" che interessa tutto il territorio rappresentato in modo piuttosto continuo, sono presenti sia una falda in pressione presente all'interno delle ghiaie di base delle alluvioni recenti, sia un sistema multifalda presente nel settore Albano dove sono individuabili almeno altre 2 circolazioni oltre quella regionale.

La prima circolazione, relativa alle vulcaniti più superficiali dell'Unità Idrogeologica dei Colli Albani, ha un andamento centrifugo rispetto all'edificio vulcanico e si raccorda con la falda sottostante a quote di circa 25 m.s.l.m.. Lungo i fianchi dell'edificio vulcanico, la circolazione viene drenata dai corsi d'acqua. La seconda può essere identificata come circolazione a carattere regionale: parte dai settori più periferici con quote attorno ai 60 m. s.l.m. e si raccorda con i livelli di base dei corsi d'acqua principali e con il mare lungo la costa. La terza ed ultima circolazione si riscontra solo al di sotto delle vulcaniti a profondità molto elevate. È caratterizzata da bassi gradienti idraulici $(10 \mathrm{~m} / \mathrm{km})$, con quote che si attestano tra $\mathrm{i}$ 15 e i 30 m s.l.m.

In sintesi quindi, il modello concettuale di circolazione dell'area romana prevede un'alimentazione di differenti falde acquifere sovrapposte (almeno 3 nel settore Albano) che dalle zone più periferiche e ad elevazione maggiore del territorio rappresentato fluiscono, raccordandosi, verso il recapito finale dei Fiumi Tevere e Aniene (Fig.2). È infatti evidente dall'assetto piezometrico descritto, come da un punto di vista generale la circolazione sotterranea segua le medesime linee di flusso della circolazione superficiale, rappresentando di conseguenza una corrispondenza piuttosto fedele tra bacini idrografici e bacini idrogeologici.

Il settore maggiormente preso in esame in questo studio, è relativo all'area Est di Roma Capitale, ovvero la superficie prevalentemente sottesa dal basso Bacino del Fiume Aniene. In questo settore, l'assetto idrogeologico (La Vigna et al. 2008, 2010) prevede importanti elementi che caratterizzano i flussi sotterranei, tra cui il Graben del Paleotevere (Luberti et al. 2017), le Sorgenti dell'Acqua Vergine, e il Fiume Aniene.

In particolare il citato Graben del Paleotevere è una depressione tettonica dentro la quale si sono accumulati sedimenti grossolani di ambiente fluviale e lacustre, per uno spessore che in alcuni punti supera gli 80 metri, che costituiscono un importante corpo acquifero (Fig.3) con uno sviluppo a direzione "appenninica" collocato sotto uno dei settori più intensamente urbanizzati di Roma.


Fig. 2 - - Hydrogeological conceptual model of Rome (Mazza et al. 2016).

Fig. 2 -Modello concettuale idrogeologico dell'area di Roma (Mazza et al. 2016). 


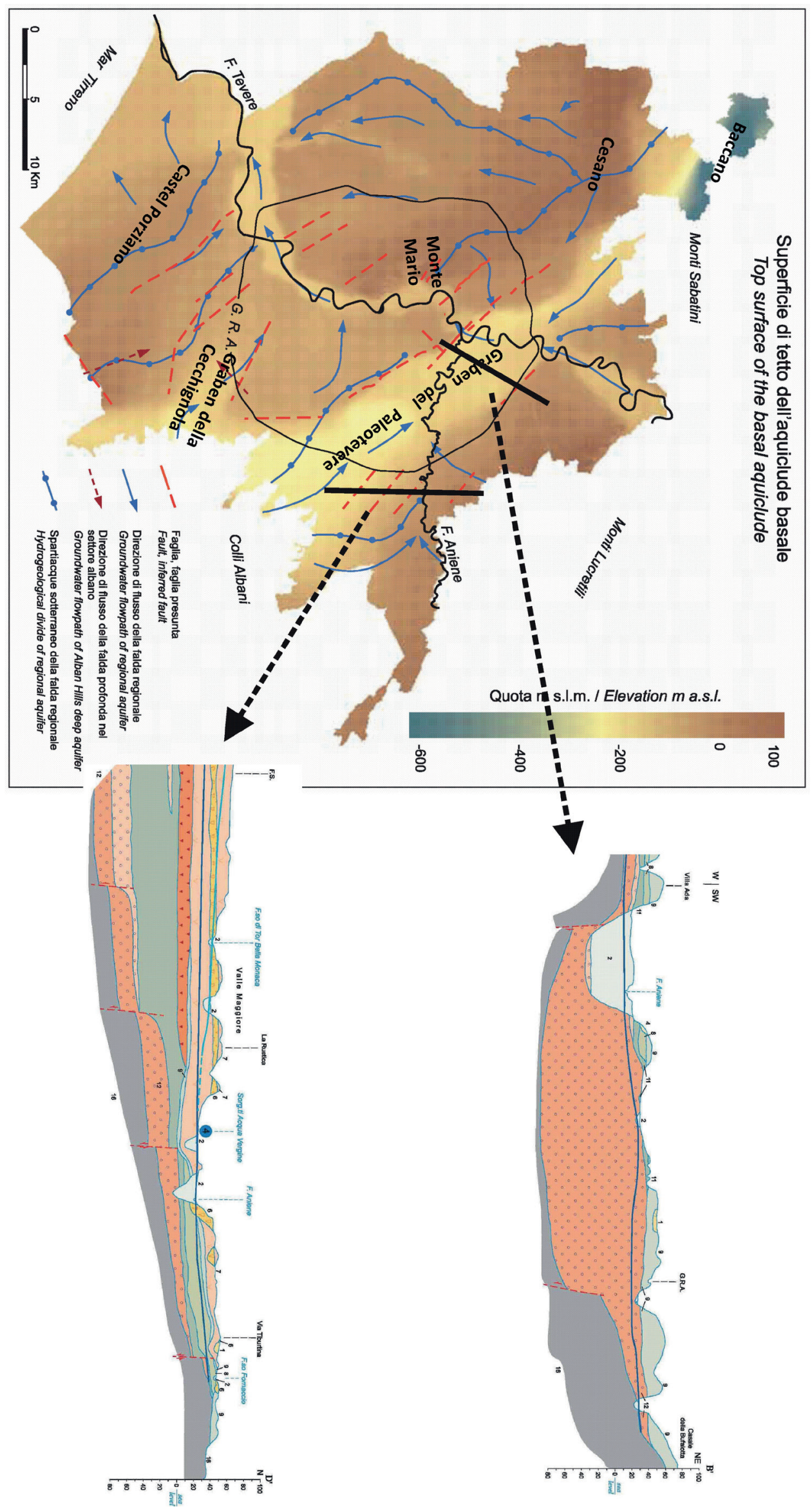




\section{Dati e metodi}

I dati trattati in questo studio sono relativi a casi notificati ai sensi del D.Lgs. 152/2006. Come accennato in introduzione la banca dati bonifiche di Roma Capitale ad oggi annovera oltre 350 siti notificati potenzialmente contaminati. Solo parte di questi presentano contaminazione accertata delle acque sotterranee, e solo una limitata percentuale ad oggi presenta contaminazione da sostanze organo alogenate (Fig. 4). In relazione a tali composti, e per il settore orientale della città, sono stati selezionati i siti dove dette sostanze sono state analizzate. In questo ambito sono state individuate 3 sottocategorie: siti con concentrazioni al di sotto del limite di rilevabilità strumentale, siti con concentrazioni al di sotto delle concentrazioni soglia di contaminazione (CSC) previste dal D.Lgs. 152/2006, e siti con concentrazioni al di sopra delle CSC.

Questi dati sono stati quindi rappresentati ed analizzati in ambiente GIS con simboli proporzionati al valore di contaminazione riscontrata. In particolare si è optato per differenziare la simbologia tra $\mathrm{i}$ casi in cui dette sostanze sono state ricercate ma non riscontrate e i casi in cui, seppure riscontrate, i valori di concentrazione siano al di sotto delle soglie stabilite dalla normativa ambientale.

\section{Risultati}

La rappresentazione della selezione dei siti con presenza accertata di solventi clorurati riporta una distribuzione prevalente nel settore orientale della città. Dei 19 siti interessati da composti organo alogenati ben 15 ricadono appunto in questo settore. Vi è poi da segnalare la situazione di presenza accertata e diffusa di clorurati nei Comuni di Pomezia ed Ardea, località subito a Sud del confine amministrativo meridionale del territorio di Roma Capitale, indicata in figura con un punto al di fuori del confine. Attualmente non sono ancora stati notificati siti nell'area limitrofa ai suddetti comuni che attestino l'eventuale migrazione di questa contaminazione diffusa anche dentro i confini amministrativi di Roma Capitale.

Rispetto alle specie rilevate sono stati rappresentati i dati di quelle maggiormente ricorrenti, ovvero Tetracloroetilene (Fig.5), Tricloroetilene (Fig.6), Triclorometano (Fig.7) e la sommatoria degli Organoalogenati (Fig.8).

Vi sono poi localmente concentrazioni anche elevate di sostanze meno ricorrenti non rappresentate in mappa. Le specie non ricorrenti ma presenti puntualmente a livelli non trascurabili sono: Cloruro di Vinile, 1,1 Dicloroetilene, 1,2 Dicloroetilene, 1,2 Dicloroetano, 1,1,2 Tricloroetano.

\section{Discussione}

La presenza di siti con superamenti relativi a sostanze organo alogenate in falda maggiormente concentrati nel settore Est di Roma può trovare una possibile spiegazione se si osserva il Piano Regolatore vigente della Città. Come si può osservare in Fig.9 infatti si nota come nel settore Est di Roma vi sia una

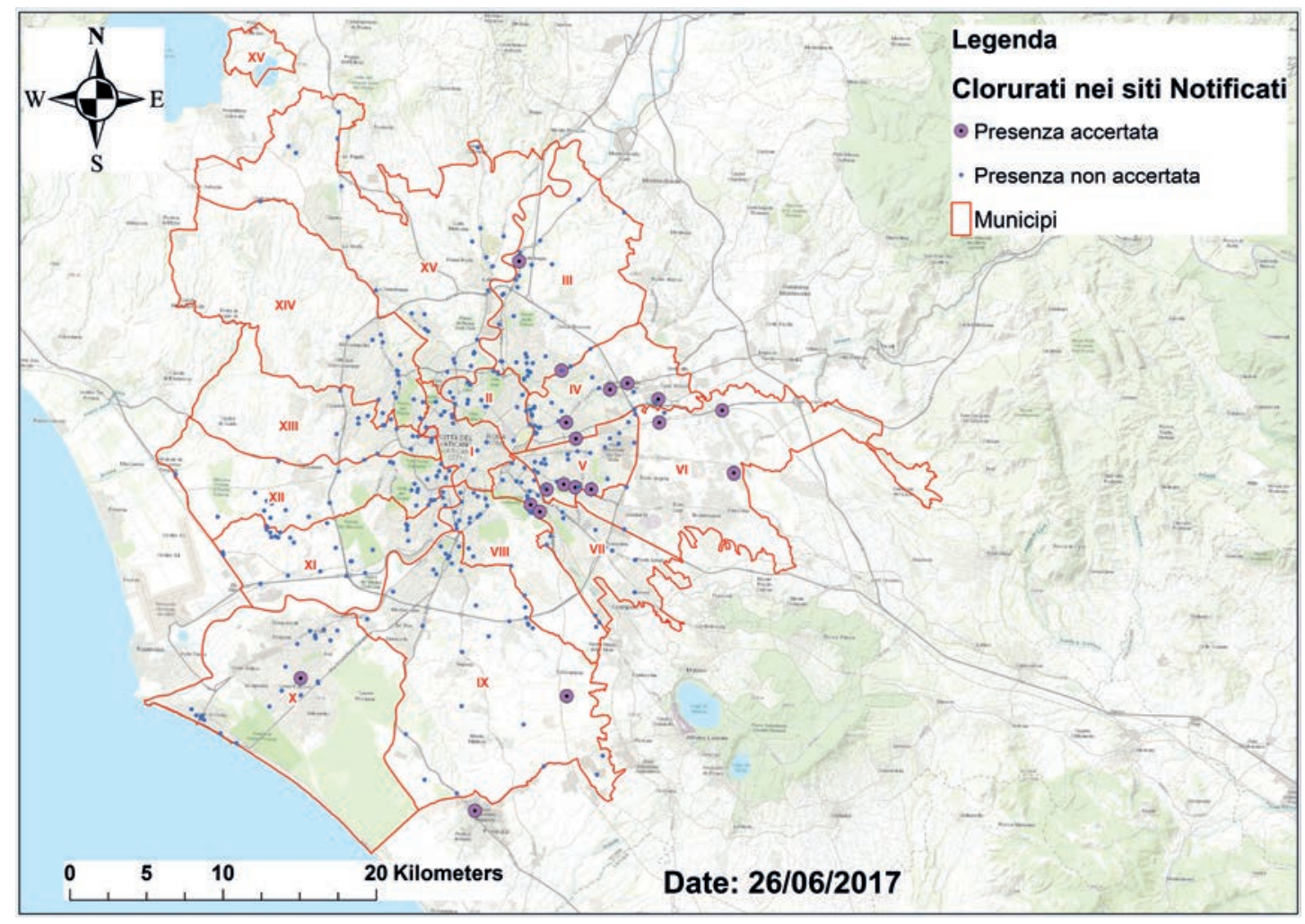

Fig. 4 - Distribution of the notified contaminated sites interested by organohalogenated compounds.

Fig. 4 - Distribuzione dei siti contaminati notificati interessati da composti organo alogenati. 


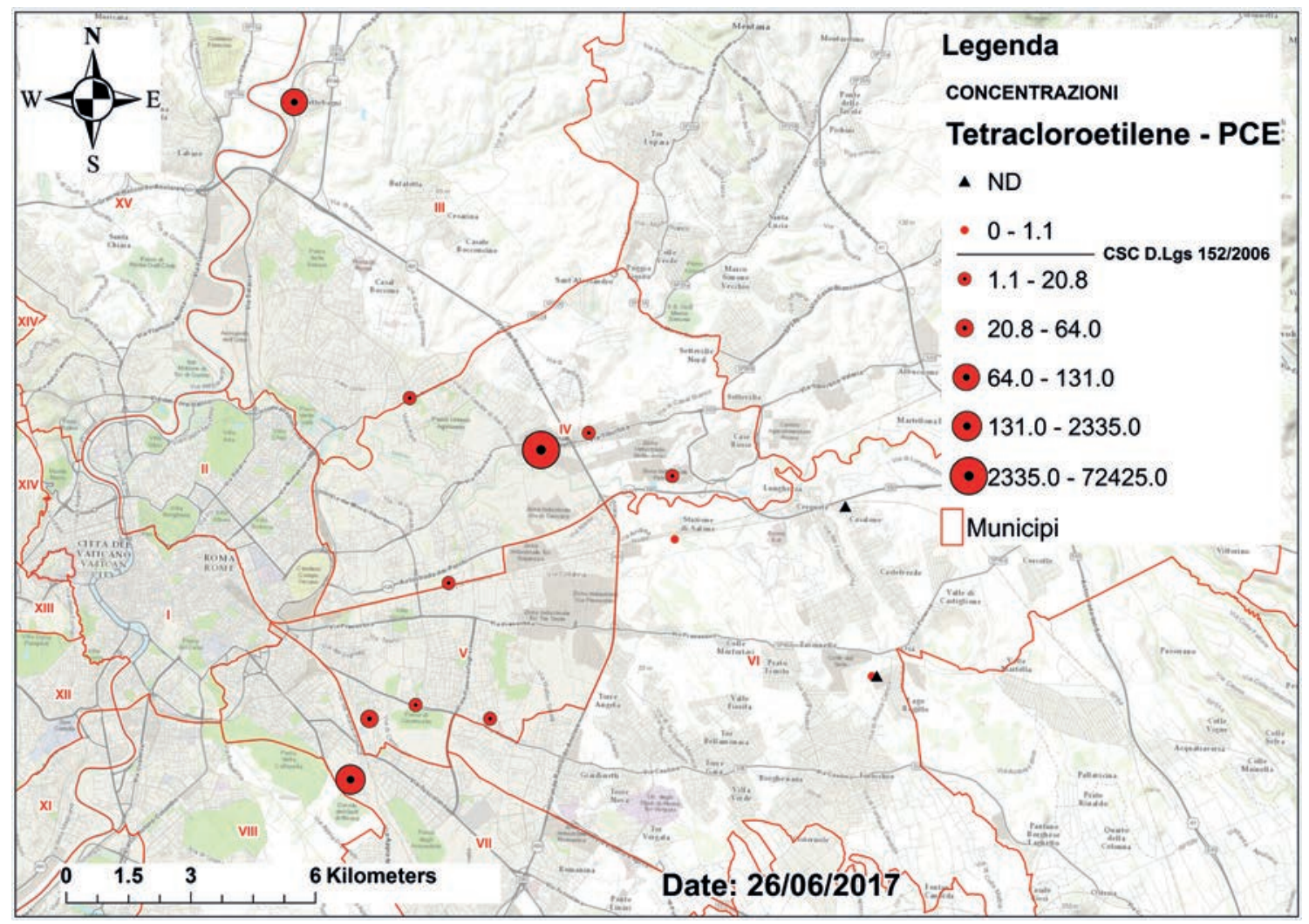

Fig. 5 - Concentration ranges of PCE ( $\mu \mathrm{g} / \mathrm{l})$ in the notified contaminated sites of Rome.

Fig. 5 - Intervallo di concentrazione del Tetracloroetilene (PCE) $(\mu \mathrm{g} / \mathrm{l})$ nei siti contaminati notificati di Roma.

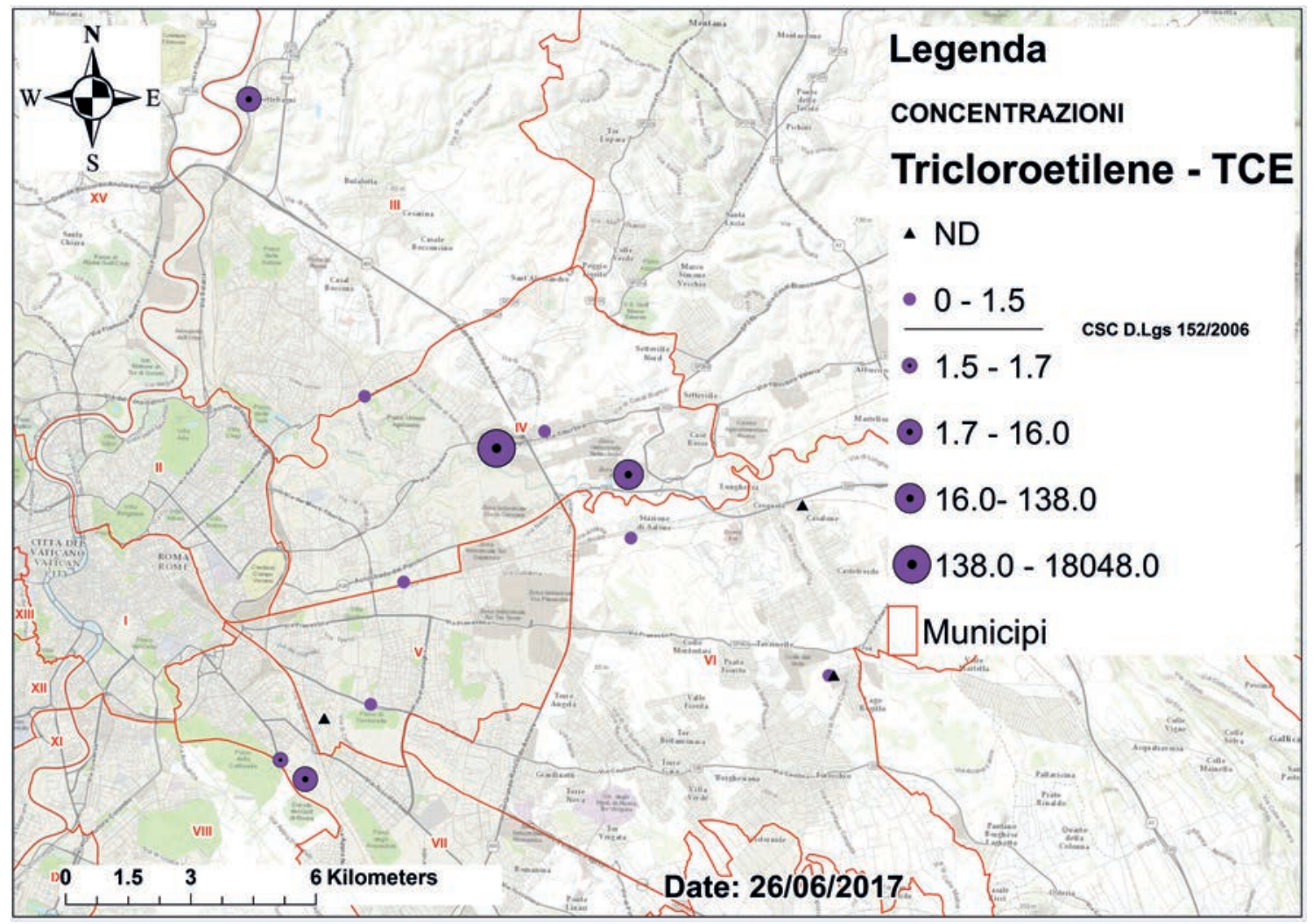

Fig. 6 - Concentration ranges of TCE ( $\mu \mathrm{g} / \mathrm{l})$ in the notified contaminated sites of Rome.

Fig. 6 - Intervallo di concentrazione del Tricloroetilene (TCE) $(\mu \mathrm{g} / \mathrm{l})$ nei siti contaminati notificati di Roma. 


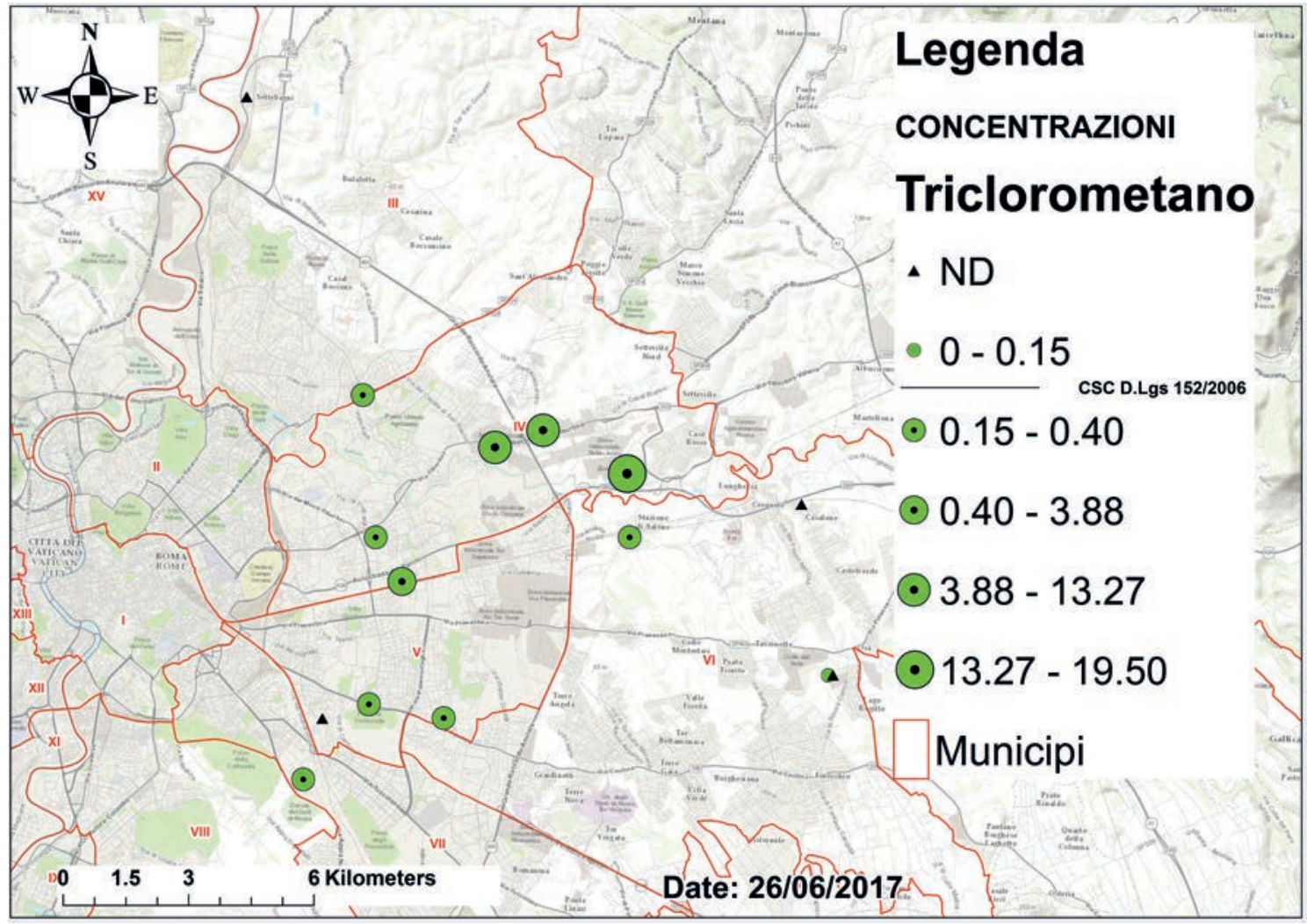

Fig. 7-Concentration ranges of Trichloromethane $(\mu \mathrm{g} / \mathrm{l})$ in the notified contaminated sites of Rome.

Fig. 7 - Intervallo di concentrazione del Triclorometano $(\mu \mathrm{g} / \mathrm{l})$ nei siti contaminati notificati di Roma.

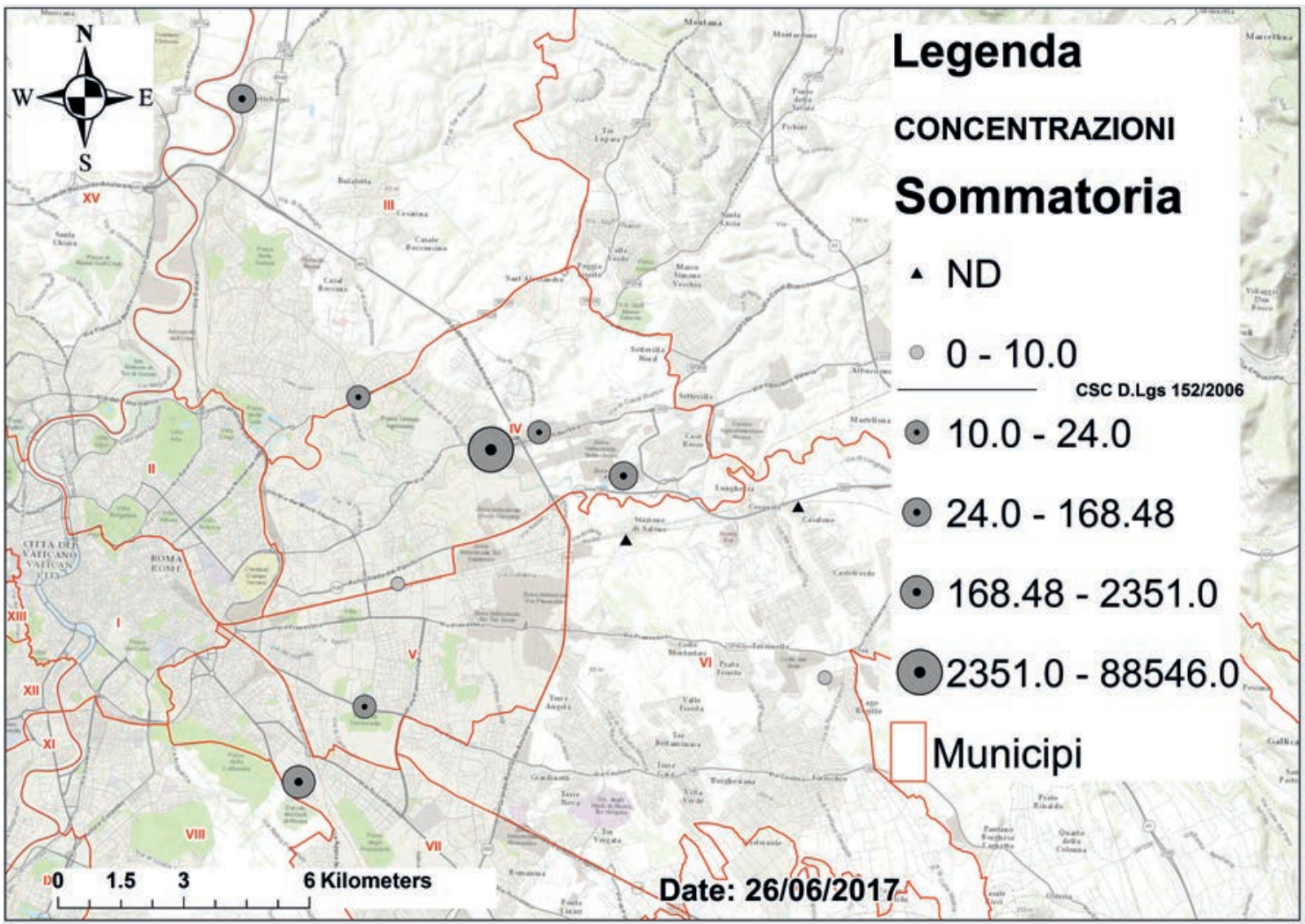

Fig. 8 - Concentration ranges of total organohalogen compound $(\mu \mathrm{g} / \mathrm{l})$ in the notified contaminated sites of Rome

Fig. 8 - Intervallo di concentrazione dei Organo alogenati totali $(\mu \mathrm{g} / \mathrm{l})$ nei siti contaminati notificati di Roma 


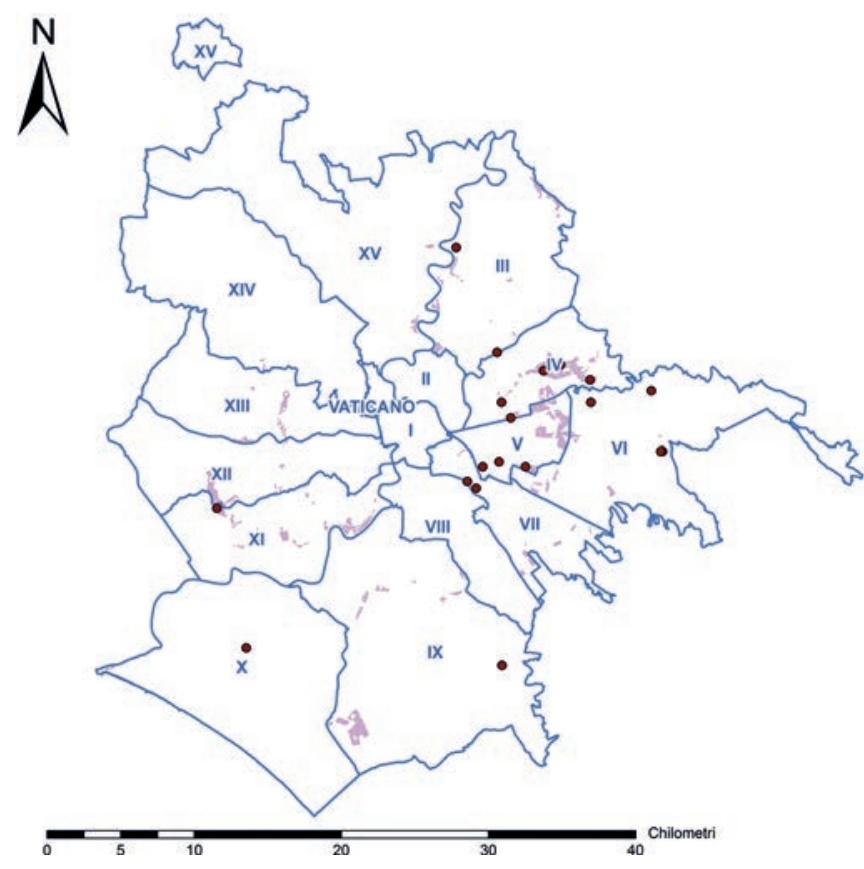

Fig. 9 - Distribution of the notified contaminated sites interested by organohalogen compound vs industrial areas of Master Plan of Rome.

Fig. 9 - Distribuzione dei siti contaminati notificati interessati da composti organo alogenati sovrapposti alle aree destinate ad impianti industriali del Piano Regolatore Generale di Roma.

discreta presenza di zone omogenee cosiddette "D", ovvero le parti di territorio destinate all'insediamento di impianti industriali. Roma storicamente non ha mai avuto una grande vocazione industriale, di conseguenza non stupisce che le contaminazioni da organo alogenati siano principalmente riscontrate al momento nel settore della città che, sebbene limitatamente rispetto ad altre realtà urbane, presenta una maggiora presenza di impianti potenzialmente "a rischio".

Le specie più comuni e maggiormente presenti nella zona

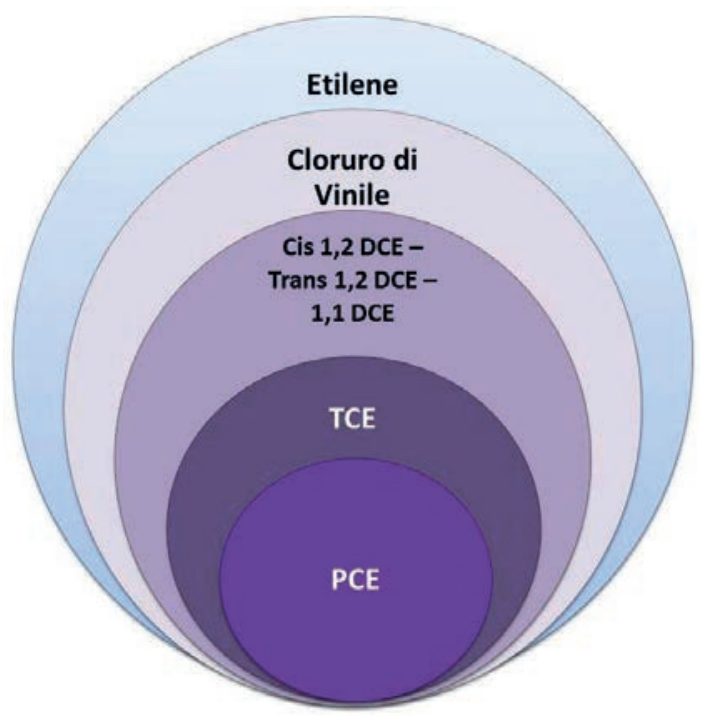

Fig. 10 - Reductive declorination process of PCE.

Fig. 10 - Processo di declorazione riduttivadel Tetracloroetilene (PCE). sono in prevalenza riconducibili alla catena di degradazione del Tetracloroetiliene (PCE) che attraverso il fenomeno della "Declorazione riduttiva" perde atomi di Cloro e si trasforma in Tricloroetilene (TCE), poi a seconda delle condizioni locali in diverse specie di Dicloroetilene (DCE), poi nel Cloruro di Vinile e successivamente nell'Etilene (Fig.10).

È importante in questi casi cercare di distinguere tra le situazioni presumibilmente riconducibili a zone molto prossime alle sorgenti puntuali (point sources - PS) dove si riscontrano valori di concentrazione elevatissimi, sicuramente riconducibili a prodotti quasi in fase libera, e le sorgenti puntuali multiple (multiple point surces - MPS), dove una serie di piccole sorgenti puntuali originarie, oggi non più definibili, distribuite in grandi aree, hanno dato nel tempo una situazione di contaminazione pressoché diffusa, caratterizzata $\mathrm{da}$ valori di contaminazione variabili, generalmente più contenuti rispetto alle situazioni di hot spots, sebbene talvolta eccedenti i limiti di legge.

Analizzando la localizzazione di questi siti del settore Est della città, e sovrapponendola all'andamento del substrato poco permeabile che costituisce l'acquicludo basale di tutta l'area romana, e di cui si è parlato in precedenza (Fig. 11), appare evidente come la maggior parte dei siti ricadano al di sopra del "Graben del Paleotevere" e dell'importante corpo acquifero in esso contenuto. Inoltre, anche se non esattamente al di sopra della struttura tettonica, molti di questi siti sono collocati in settori in cui il deflusso sotterraneo, evidenziato con le linee piezometriche relative all'acquifero regionale (Fig. 11), si dirige comunque verso la medesima depressione. Sulla base delle caratteristiche di questi inquinanti e della loro capacità, in fase separata, di penetrare a fondo nelle falde per via della loro densità maggiore dell'acqua, la loro presenza in questi settori caratterizzati dall'assetto idrogeologico precedentemente descritto, rappresenta seri rischi di dispersione nelle porzioni più profonde di acquifero. Sebbene Roma sia principalmente servita da acque provenienti dal vicino appennino e solo in parte da captazioni degli acquiferi vulcanici dei Colli Albani, su cui tali rischi potrebbero insistere nell'immediato futuro, nella città sono presenti svariate centinaia di pozzi che attingono dai vari corpi acquiferi per usi industriali, commerciali e domestici.

A tal riguardo la presenza di pozzi di proprietà dell'Amministrazione Capitolina distribuiti in maniera diffusa sul territorio, e che a seguito di una attività di rilevamento e censimento recentemente svolta, costituiscono la "Rete di Monitoraggio delle Acque Sotterranee di Roma Capitale" (La Vigna et al 2015), potrà sicuramente rappresentare un utile elemento per approfondimenti specifici sul tema.

\section{Conclusioni}

Si è visto come anche a Roma, città dalla limitata vocazione industriale, il problema della contaminazione delle acque di falda da solventi clorurati sia una questione emergente, specialmente in quei settori dove si sono insediati i pochi stabilimenti industriali romani. La maggior parte di questi siti è stata notificata ai sensi dell'art. 245 del D.Lgs. 


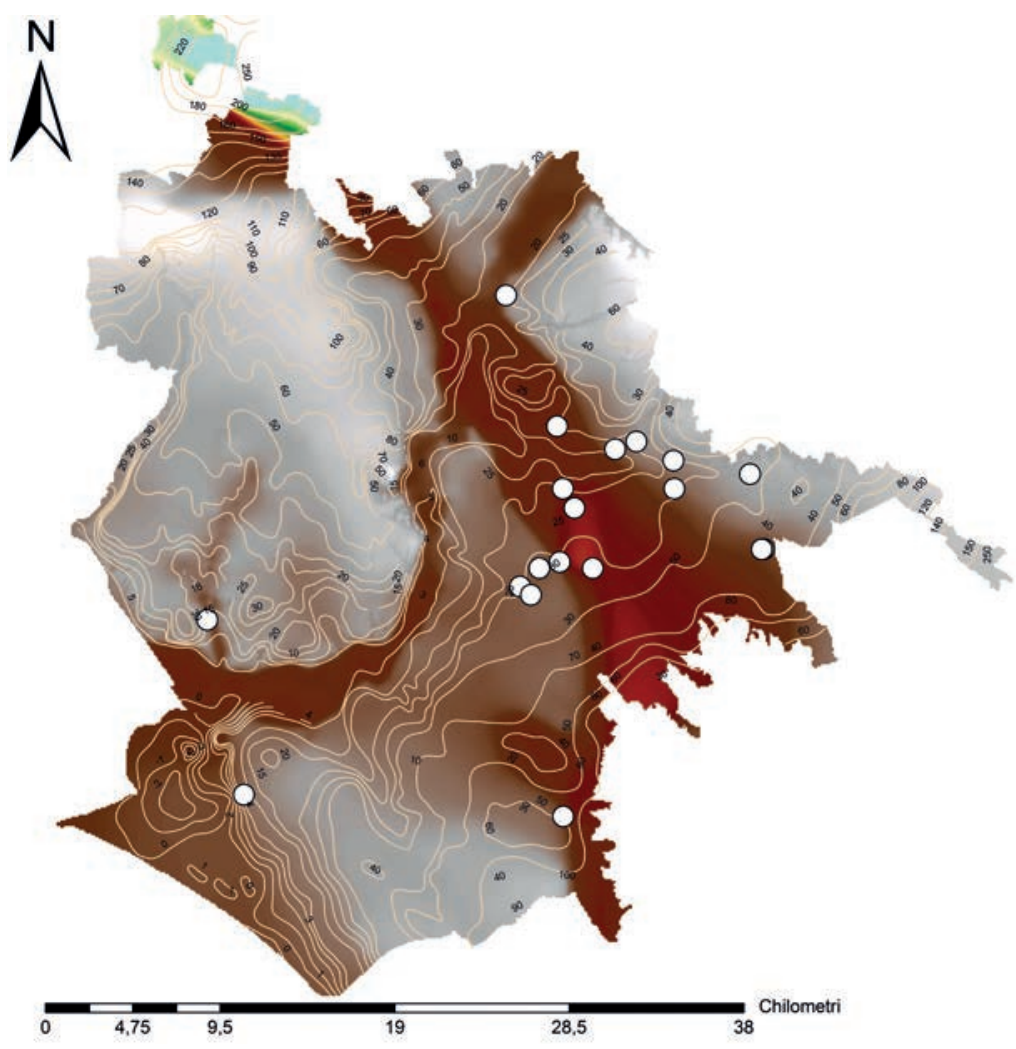

Fig. 11 - Distribution of the sites interested by organobalogenated compounds vs basal aquiclude surface and regional water table. The deepest zone of the Paleotiber Graben structure (brown NW-SE oriented structure) could be potentially affected by this pollution due to location and to main groundwater flowpath.

Fig. 11 - Distribuzione dei siti interessati da composti organo alogenati sovrapposti all'andamento della superficie di tetto dell'acquicludo basale e alla piezometria dell'acquifero regionale. La zona più profonda del Graben del Paleotevere risulta potenzialmente interessata alla migrazione della contaminazione sia per ubicazione che per destinazione delle linee di flusso sotterraneo.
$152 / 2006$, ovvero in qualità di "soggetto non responsabile" della contaminazione. Questo comporta che sia la Città Metropolitana di Roma Capitale, ente preposto alle indagini, a dover individuare eventuali responsabilità. L'esperienza delle altre realtà urbane italiane insegna però che, fatti salvi i casi in cui le evidenze siano tali da rendere possibile l'accertamento delle responsabilità, nella maggior parte delle situazioni le Amministrazioni regionali devono intraprendere un complesso percorso amministrativo, inter-istituzionale, volto alla predisposizione di piani per l'inquinamento diffuso. A tal riguardo, solo di recente il Sistema Nazionale per la Protezione dell'Ambiente ha predisposto il documento "Criteri per la elaborazione di piani di gestione dell'inquinamento diffuso" (Bondi et al 2016), al fine di delineare in modo omogeneo procedure per la redazione di questi piani.
Certamente la predisposizione dei suddetti piani richiede l'impiego di ingenti risorse da parte delle Amministrazioni e lunghi tempi di esecuzione. Inoltre, nel caso di contaminazione da sostanze organo alogenate, laddove queste abbiano determinato plumes estesi, con mobilità a volte elevata e lunga persistenza anche nelle porzioni profonde degli acquiferi, le reali possibilità di pervenire ad una completa bonifica a costi sostenibili su larga scala possono risultare limitate.

Va sottolineato che, per alcuni composti, i limiti previsti dalla normativa ambientale D.Lgs. 152/2006, sono più restrittivi di quelli previsti dal D.Lgs. 31/2001 (Attuazione della direttiva 98/83/CE relativa alla qualità delle acque destinate al consumo umano), come sintetizzato nella successiva Tab.1 dalla quale si evince che possano essere considerate "potabili" per il D.Lgs. 31/2001, acque contaminate secondo il D.Lgs.152/2006.

Tab. 1 - Comparison between concentration limits of some organohalogenated compounds in water according to Italian laws D.lgs. $152 / 2006$ (environment main law) and D.Lgs. 31/2001 (regulation related to water for human consumption).

Tab. 1 - Confronto tra i limiti di concentrazione di alcune specie di composti organo alogenati nelle acque secondo le normative italiane D.lgs. $152 / 2006$ (Codice dell'Ambiente) e D.Lgs. 31/2001 (regolamentazione acque destinate al consumo umano).

\begin{tabular}{|l|c|c|}
\hline Nome Specie - Compound & D.Lgs. 152/2006 & D.Lgs. 31/2001 \\
\cline { 1 - 2 } Tetracloroetilene (PCE) & $1.5 \mu \mathrm{g} / \mathrm{l}$ & $\begin{array}{c}\text { Sommatoria } \\
10 \mu \mathrm{g} / \mathrm{l}\end{array}$ \\
\hline Tricloroetilene (TCE) & $1.1 \mu \mathrm{g} / \mathrm{l}$ & \multirow{2}{*}{$\begin{array}{c}\text { Sommatoria } \\
30 \mu \mathrm{g} / \mathrm{l}\end{array}$} \\
\hline Triclorometano (Cloroformio) & $0.15 \mu \mathrm{g} / \mathrm{l}$ & \\
\hline Tribromometano (Bromoformio) & $0.3 \mu \mathrm{g} / 1$ & $0.13 \mu \mathrm{g} / \mathrm{l}$ \\
\cline { 1 - 2 } Dibromoclorometano & $0.17 \mu \mathrm{g} / \mathrm{l}$ & \\
\hline Bromodiclorometano & & \\
\hline
\end{tabular}


Per quanto concerne la possibilità di utilizzo per altri scopi, quali quelli agricoli, di acque contenenti le sostanze in argomento, si richiama il D.M. n.185 del 12/6/2003 (Regolamento recante norme tecniche per il riutilizzo delle acque reflue in attuazione dell'articolo 26, comma 2, del D.Lgs. 11 maggio 1999, n. 152). In tale normativa il limite fissato per i solventi clorurati è, in analogia alla normativa per le acque potabili, di $10 \mu \mathrm{g} / \mathrm{l}$ per la sommatoria tra Tetracloretilene e Tricloroetilene e di $30 \mu \mathrm{g} / \mathrm{l}$ per la sommatoria dei Trialometani, mentre il limite per la sommatoria dei Solventi clorurati è fissato in $40 \mu \mathrm{g} / \mathrm{l}$.

Circa il rischio associato al consumo di ortaggi e specie vegetali irrigati con acque contenenti solventi clorurati, a titolo di esempio, si citano alcuni autori che hanno testato l'assorbimento di Tricloroetilene (Chard et al. 2006) su alberi da frutta (meli e peschi), i quali concludono che il principale assorbimento risulterebbe avvenire nelle radici, nel tronco e nei rami, mentre nelle foglie e nei frutti non sarebbe stata rilevata la presenza di questa sostanza.

Risulta per quanto sopra evidente la necessità di approfondimenti e studi che possano consentire la redazione di linee guida ed indicazioni sul tema da parte delle autorità sanitarie nazionali, vista anche la crescente richiesta di rassicurazioni da parte della cittadinanza.

Nel merito si evidenzia che in assenza di tali approfondimenti, l'esigenza della Pubblica Amministrazione di attenersi al principio di precauzione, si traduce sovente in misure restrittive e limitazioni d'uso, con il paradosso, nel caso frequente di lieve contaminazione, di interdire l'utilizzo o imporre sistemi di trattamento anche per acque che avrebbero caratteristiche di potabilità.

Ringraziamenti: Si ringraziano le tesiste Lucrezia Aleotti e Azzurra Covarelli, dell'Università La Sapienza di Roma, per la collaborazione nella sintesi dei dati presenti nei nostri archivi.

\section{BIBLIOGRAFIA}

Alberti L, Colombo L, Formentin G (2017) A comparison between two stochastic approaches to assess groundwater PCE diffuse pollution in Milano functional urban area. Proceedings of Flowpath2017 National Meeting on Hydrogeology. Cagliari

Bondi C, Giandon P, Guerra M, Calace N, Doni A, Simonetto F, Grillo A, Iorio R, Ferri D, Racciatti RV, Perfumi P, Rossanigo P, Fogal E, Catalano L, Dessì R, Arena A, Marzocchini M, Desiderio G (2016) Criteri per la elaborazione di piani di gestione dell'inquinamento diffuso "Criteria for the development of widespread pollution management plans". Sistema Nazionale per la Protezione dell'Ambiente.

Busoni S, Gnocchi A (2013) Problematiche nella contaminazione delle falde da solventi clorurati. Esperienze in Provincia di Treviso "Problems of contamination of groundwater with solvents. Experiences in the Province of Treviso". La bonifica dei siti contaminati in Regione del Veneto. Padova, 22 Marzo 2013.

Chard BK, Doucette WJ, Chard JK, Bugbee B, Gorder K (2006) Trichloroethylene uptake by apple and peach trees and transfer to fruit. Environmental Science and Technology 40:15. DOI:10.1021/es060156k

Dimasi M, Mancini M, Mazza R (2015) Superficie di tetto dell'Aquiclude basale "Top Surface of the basal Aquiclude", in La Vigna F and Mazza R (Eds.) Carta Idrogeologica di Roma "Hydrogeological Map of Rome" - Scala/Scale 1:50.000

Ellis B (1999) Impacts of urban growth on surface water and groundwater quality. IAHS no. 259, IAHS, Wallingford, UK, 437 pp

Verdini R, Majone M (2015) Presenza e comportamento dei Solventi Clorurati "Presence and and behavior of chlorinated solvents", in Pinna $\mathrm{P}$ and Riva M (Ed.) Analisi e promozione di nuove tecnologie di bonifica e di caratterizzazione dei siti "Analysis and promotion of new remediation and characterization technologies for contaminated sites”, Éupolis Lombardia CODICE: TER 13010/001

Guzzella L, Salerno F, Ravelli S, D'ambrosio G (2009) Lo stato qualitativo dei corpi idrici sotterranei in Provincia Di Milano: Rapporto Finale "Groundwater quality status in the province of Milano". Rapporto Di Ricerca Irsa-Cnr/Provincia Di Milano - 107 PP., Brugherio, Milano - Ed. IRSA-CNR

Howard KWC, Israfilow R Eds. (2002) Current Problems of Hydrogeology in Urban Areas, Urban Agglomerates and Industrial Centres. NATO Sciences Series IV. Earth and Environmental Sciences.8. Springer-Science+Business Media, B.V. DOI 10.1007/978-94-010-0409-1

La Vigna F, Mazza R, Amanti M, Di Salvo C, Petitta M, Pizzino L (2016) The synthesis of decades of groundwater knowledge: the new Hydrogeological Map of Rome. Acque Sotterranee - Italian Journal of Groundwater: 4/142 DOI 10.7343/as-128-15-0155

La Vigna F, Mazza R Eds (2015) Carta Idrogeologica di Roma "Hydrogeological Map of Rome" Scala/Scale 1:50.000. Roma Capitale

La Vigna F, Bonfa I, Martelli S (2015) The Groundwater Monitoring Network of Rome. AQUA2015 Hydrogeology back to the future! $42^{\circ}$ IAH Congress, Rome 13-18 September

La Vigna F, Bonfa I, Martelli S (2014) La determinazione dei valori di fondo naturale ed antropico nelle acque sotterranee dei grandi agglomerati urbani "Detection of natural and anthropic background values in the underground waters of large urban agglomerations". Acque Sotterranee - Italian Journal of Groundwater: 3: 2/136 DOI 10.7343/AS-074-14-0100

La Vigna F, Ciadamidaro, S., Mazza, R. \& Mancini, L. (2010) Water quality and relationship between superficial and ground water in Rome (Aniene River basin, central Italy). Environmental Earth Sciences, 60(6), DOI 10.1007/s12665-009-0267-2 
La Vigna, F., Capelli, G. \& Mazza, R. (2008) Carta Idrogeologica del Settore Terminale del Bacino del Fiume Aniene (Roma) "Hydrogeological Map of the Lower Aniene River Basin". In Funiciello, R., Giordano, G. \& Mattei M. (Eds) Mem. Descr. Carta Geol. d'It., 80, Roma - ISSN -0242

Luberti GM, Marra F, Florindo F (2017) A review of the stratigraphy of Rome (Italy) according to geochronologically and paleomegnetically constrained aggradational successions, glacio-eustatic forcing and volcano-tectonic processes. Quaternary International: 438 DOI: 10.1016/j.quaint.2017.01.044

Mazza R, La Vigna F, Capelli G, Dimasi M, Mancini M, Mastrorillo L (2016) Idrogeologia del territorio di Roma "Hydrogeology of Rome". Acque Sotterranee - Italian Journal of Groundwater: 4/142 DOI10.7343/as-129-15-0156

Menichetti S, Doni A (2017) Organohalogen diffuse contamination in Firenze and Prato groundwater bodies. Investigative monitoring and definition of background values. Acque Sotterranee - Italian Journal of Groundwater: 6 : 1/147 DOI 10.7343/as-2017-260
Pedretti D, Masetti M, Beretta GP, Vitiello M (2013) A Revised Conceptual Model to Reproduce the Distribution of Chlorinated Solvents in the Rho Aquifer (Italy). GroundWater Monit R, 33 : 69-77. Doi:10.1111/gwmr.12017

Preziosi E, Giuliano G, Vivona R (2009) Natural background levels and threshold values derivation for naturally As, $\mathrm{V}$ and $\mathrm{F}$ rich groundwater bodies: a methodological case study in Central Italy. Environmental Earth Science. DOI 10.1007/s12665-009-0404-y

Vanzetti C, Gianoglio N, Sesia E (2016) Studio sulla contaminazione diffusa da solventi clorurati nelle acque sotterranee "Study on diffuse contamination of chlorinated solvents in groundwater". Arpa Piemonte, Struttura Specialistica Qualità delle Acque

Vazquèz-Suňè E, Sànchez-Vila X, Carrera J (2005) Introductory review of specific factors influencing urban groundwater, an emerging branch of hydrogeology, with reference to Barcelona, Spain. Hydrogeology Journal 13:522-533. DOI 10.1007/s10040-004-0360-2

Ventriglia U (1971) La Geologia della Città di Roma "The geology of Rome". Amm. Prov. di Roma

Ventriglia U (2002) Geologia del territorio del Comune di Roma "Geology of Rome municipality". Amm. Prov. di Roma 